\title{
Association of Short and Long Interpregnancy Intervals with Adverse Birth Outcomes: Evidence from a Cross-Sectional Study in Northwest China
}

\author{
Guoshuai Shi' \\ Binyan Zhang' \\ Yijun Kang' \\ Shaonong Dang $\mathbb{D}^{\prime}$ \\ Hong Yan $^{1,2}$ \\ 'Department of Epidemiology and \\ Biostatistics, School of Public Health, \\ Xi'an Jiaotong University Health Science \\ Center, Xi'an, Shaanxi, 71006I, People's \\ Republic of China; ${ }^{2}$ Nutrition and Food \\ Safety Engineering Research Center of \\ Shaanxi Province, Xi'an, Shaanxi, 7I006I, \\ People's Republic of China
}

Purpose: To analyze the effects of a short interpregnancy interval (IPI) ( $<6$ months) and a long IPI ( $>120$ months) on neonatal adverse birth outcomes including low birth weight (LBW), small for gestational age (SGA), preterm birth (PTB), and birth defects in Shaanxi Province.

Patients and Methods: A stratified multistage random sampling method was used to recruit participants who gave birth between 2010 and 2013 in Shaanxi province. A self-designed questionnaire was used to collect the information of the participants. With the confounding factors controlled, the generalized linear model (GLM) was used to investigate the association between IPI and neonatal birth outcomes. The restricted cubic spline (RCS) function was used to evaluate the dose-response relationship between IPI and birth outcomes.

Results: A total of 13,231 women were included. The prevalence of LBW, SGA, PTB, and birth defects was $3.24 \%, 12.96 \%, 2.93 \%$, and $2.12 \%$, respectively. GLM showed that a short IPI ( $<6$ months) was associated with a higher risk of SGA $(\mathrm{RR}=1.25,95 \%$ CI: $1.04-1.52)$ and birth defects $(\mathrm{RR}=2.55,95 \% \mathrm{CI}$ : $1.45-4.47)$, and a long IPI ( $\geq 120$ months) was associated with a higher risk of $\mathrm{LBW}(\mathrm{RR}=1.54,95 \% \mathrm{CI}: 1.01-2.34)$ and $\mathrm{PTB}(\mathrm{RR}=1.73$, 95\% CI: 1.08-2.76) than an IPI of 18-23 months. The RCS showed that LBW, SGA, and PTB demonstrated a $j$-shaped relationship with IPI ( $P$ for overall association $<0.001$ for these three birth outcomes), and birth defects ( $\mathrm{P}$ for overall association $<0.001$ ) had an inversely non-linear relationship with IPI.

Conclusion: Both short and long IPIs are associated with an increased risk of adverse birth outcomes.

Keywords: interpregnancy interval, low birth weight, small for gestational age, preterm birth, birth defects

\section{Introduction}

Birth outcomes are critical indicators for measuring infant health and predicting infant growth and development. ${ }^{1}$ Adverse birth outcomes such as low birthweight (LBW), small for gestational age (SGA), preterm birth (PTB), and birth defects are important public health problems globally. ${ }^{2,3}$ PTB is the leading cause of neonatal death and the second leading cause of death among children under 5 years old. ${ }^{4}$ LBW is associated with a high risk of perinatal mortality and contributes to up to $80 \%$ of neonatal mortality. ${ }^{5}$ Birth defects and SGA increase the risks of early miscarriage, stillbirth, perinatal death, infant death, and congenital disabilities. ${ }^{6,7}$ In addition, adverse birth outcomes are also related to subsequent health problems in offspring. ${ }^{8}$ Hence, it is essential to identify the risk factors for adverse birth outcomes.
Correspondence: Shaonong Dang; Hong Yan Department of Epidemiology and Biostatistics, School of Public Health, Xi'an Jiaotong University Health Science Center, Xi'an, Shaanxi, 7 1006I, People's Republic of Chin

Email tjdshn@mail.xjtu.edu.cn; xjtu_yhpaper@I26.com 
Several interventions that may affect adverse pregnancy outcomes, such as interpregnancy interval (IPI), ${ }^{9}$ have been proposed. IPI has been identified as a key and a potentially modifiable risk factor for adverse birth outcomes. ${ }^{10-12}$ IPI refers to the interval between the previous delivery date and the conception date of the present pregnancy. ${ }^{13}$ The World Health Organization (WHO) emphasized the necessity of investigating the impact of IPI on adverse birth outcomes in future studies. ${ }^{14}$ Studies have found that the incidence of SGA, PTB, LBW, and birth defects all have $\mathrm{j}$-shaped relationships with IPI.9 Compared with an IPI of $18-23$ months, short $(<18$ months) and long ( $>23$ months) IPIs are associated with a higher risk of adverse birth outcomes. ${ }^{15}$ In addition, several studies have demonstrated that a shorter IPI $(<6$ months) is associated with an increased risk of adverse pregnancy outcomes. ${ }^{12,16}$ Based on this evidence, public health guidelines and clinical postpartum best practices recommend a minimum interval of 18-24 months between births. ${ }^{12,17}$ Despite several studies on the association between a shorter IPI ( $<6$ months) and adverse pregnancy outcomes, there are limited studies on this issue involving the Chinese population. Some studies have also reported that a longer IPI (>120 months) may lead to adverse birth outcomes. ${ }^{13}$ However, only a few studies are investigating the effect of longer IPIs ( $>120$ months) on pregnancy outcomes, and no specific conclusions can be drawn.

Therefore, the present study aimed to overcome these shortcomings. This study employed data from a large-scale population survey conducted in Shaanxi Province to analyze the association of short and long IPIs with adverse birth outcomes, and provided a theoretical basis for the formulation of government public health policies and the guidance of reproductive women in this region.

\section{Patients and Methods}

\section{Study Design and Participants}

The data used in this study were obtained from a crosssectional survey carried out in Shaanxi Province of Northwest China from August to December 2013 aiming to investigate the prevalence and risk factors of adverse birth outcomes. According to the urban-rural population ratio and fertility rate, this research adopted a stratified multistage random sampling method. First, 10 districts and 20 counties were randomly selected from urban and rural areas, respectively. In the urban area, 3 streets were randomly selected from each chosen district, 6 communities were randomly selected from each chosen street, and 60 eligible women were randomly selected from each chosen community. In the rural area, 6 townships were randomly selected from each sample county, 6 villages were randomly selected from each sample township, and 30 eligible women were randomly selected from each sample village. The inclusion criteria in this study included women of childbearing age (16-49 years) with a history of pregnancy between 2010 and 2013 and specific pregnancy outcomes and multiparous women with parities of $\geq 2$. The exclusion criteria included having severe diseases (such as cardiovascular disease or cancer) during the investigation, non-live births, primiparas, unknown birth history, multiple pregnancies, and missing birth outcomes.

After obtaining written informed consent, specially trained investigators used a structured questionnaire designed by the School of Public Health of Xi'an Jiaotong University to conduct face-to-face interviews and collect data from the respondents. To ensure the accuracy of the data, the supervisor checked all questionnaires to see if there were any missing values or logical errors. If any problems were found, the questionnaire was reinvestigated. After the survey in each district (county), $5 \%$ of the survey participants were randomly selected for repeated surveys to ensure the authenticity and credibility of the data.

A total of 32,400 pregnant women were invited to partake in the survey, and 30,027 people completed the questionnaire, with a response rate of $92.7 \%$. We further excluded 16,796 women for the following reasons: nonlive births for the latest pregnancy $(\mathrm{n}=761)$, primiparas $(n=14,402)$, unknown birth history $(n=1218)$ and missing birth outcomes for the latest pregnancy $(n=415)$. Finally, 13,231 women with an IPI were selected for the analysis. The sampling strategy with the exclusion criteria in this study is presented in Figure 1.

\section{Ascertainment of Study Variables}

IPI was used as the exposure variable in this study. The period between two consecutive deliveries was used to calculate the IPI by subtracting the gestational week of the second pregnancy. The gestational age was a measure of the age of a pregnancy. It was determined based on the last menstrual period, verified by ultrasound examination, and self-reported by participants in week. IPI was measured in months. Thirteen weeks corresponded to 3 months for the gestational week. ${ }^{18}$ In the study, IPI was modeled as a classified variable to facilitate a better understanding 


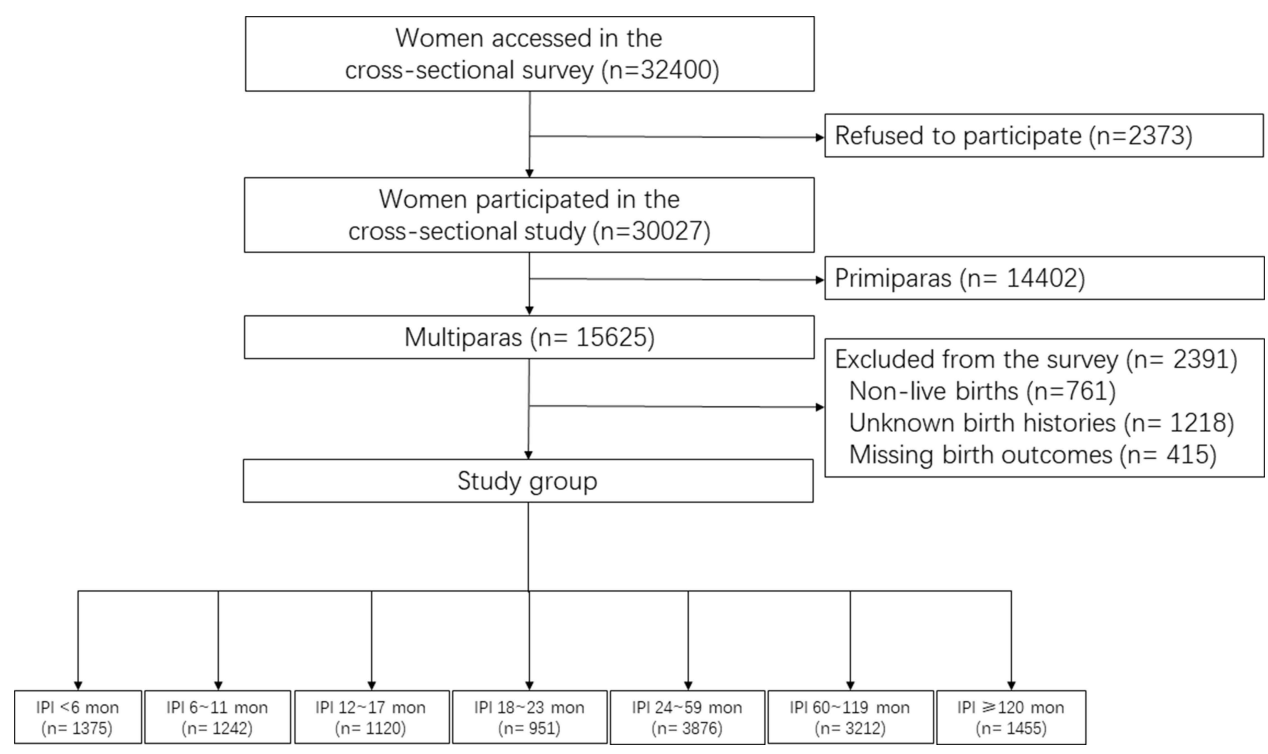

Figure I Study flow chart of sampling strategy.

of the associations between shorter or longer IPIs and adverse pregnancy outcomes and continuous variables to show the dose-response relationship. Following previous studies, ${ }^{19,20}$ IPI was divided into 7 groups $(<6,6-11,12$ $17,18-23,24-59,60-119$ and $\geq 120$ months) and an IPI of 18-23 months was set as the reference category.

The adverse pregnancy outcomes, which were the dependent variables, in this study included LBW, SGA, PTB, and birth defects. Birth weight was obtained from the medical birth certificate. Within 1 hour after delivery, birth weight was measured with a baby scale with precision to the nearest 10 grams. LBW was defined as a birth weight $<2500 \mathrm{~g}$. Gestational age was calculated in weeks based on the last menstrual period and the birth date. PTB was defined as birth within less than 37 gestational weeks. SGA was defined as a birth weight of $<10$ th percentile according to the gestational age-sex specific Chinese reference for fetal growth. ${ }^{21}$ Information on birth defects was collected using a structured questionnaire according to ICD-10 (international classification of diseases). Eleven different groups of birth defects were collected in this study, and they included those affecting the nervous system (Q00-Q07), eye, ear, face and neck (Q10-Q18), cardiovascular system (Q20-Q28), respiratory system (Q30Q34), oral clefts (Q35-Q37), digestive system (Q38-Q45), genital organs (Q50-Q56), urinary system (Q60-Q64), musculoskeletal system (Q65-Q79), other defects (Q80 Q89), and chromosomal abnormalities (Q90-Q99). ${ }^{22}$

\section{Ascertainment of Covariates}

Several factors have been associated with adverse pregnancy outcomes based on previous studies. ${ }^{23-25}$ These factors can confound the relationship between the IPI and adverse pregnancy outcomes and were therefore adjusted in this study. They were as follows: childbearing age $(<25,25-29,30-34$, or $\geq 35$ years $)$; maternal education (primary school or lower, secondary school or senior high school or higher); residence (urban or rural); family economic status (low, middle or high); maternal occupation (peasant/housework or other); passive smoking (yes or no); folic acid supplement (yes or no); iron supplement (yes or no); frequency of antenatal care visits $(\geq 5$ or $<5)$; taking medicine during maternity period (yes or no); and infant gender (male or female). The per capita annual household income was calculated by dividing the annual household income by the number of family-adjusted adults. ${ }^{26}$ The number of familyadjusted adults was calculated by adding 0.5 times the number of children to the number of adults. According to the quartile of the per capita annual household income, the family economic statuses were categorized into three: low, middle, or high. Passive smoking was defined as inhaling tobacco smoke from others for more than $15 \mathrm{~min} /$ day. $^{27}$ Folic acid supplementation was defined as taking folic acid for more than 30 days during pregnancy. ${ }^{28}$ We obtained information on covariates from the baseline questionnaire. 


\section{Statistical Analysis}

The characteristics of the participants were described. The continuous variables were expressed as mean $\pm \mathrm{SD}$ or median (interquartile range). The categorical variables were expressed as number (percentage), and the chi-squared test $(\chi 2)$ was used to compare the groups. We used the generalized linear model (GLM, binomial distribution with the log-link function) to estimate the RR and the $95 \% \mathrm{CI}$ for adverse birth outcomes (LBW, SGA, PTB, and birth defects) associated with IPI. IPI was used as a classification variable in this model for more significant effects. We established two models to control the confounders step by step: (1) model 1 was unadjusted for any covariates; (2) model 2 was adjusted for all the potential confounders mentioned above (childbearing age, maternal education, residence, family economic status, maternal occupation, passive smoking, folic acid supplement, iron supplement, frequency of antenatal care visits, taking medicine during maternity, and infant gender). Moreover, a restricted cubic spline (RCS) function was used to evaluate the doseresponse relationship between the IPI and the adverse pregnancy outcomes, using IPI as a continuous variable. In this model, four knots located at the 25th, 50th, 75th and 95th percentiles of the IPI were set, and an IPI of 18 months was set as the reference value. The P-value for the overall association was used to assess the overall association between IPIs and adverse birth outcomes, and the P-value for the nonlinear association was used to assess any nonlinear association between IPIs and adverse birth outcomes. Due to the complex design, weighting would be used to the analysis by prefixing commands with "svy" in STATA. Finally, to further examine the robustness of the effect of IPI on adverse birth outcomes, a sensitivity analysis was conducted as part of a subgroup analysis based on maternal age ( $<35$ or $\geq 35$ years) and iron supplement (yes or no) with all covariates adjusted.

EpiData 3.1 (CDC, Atlanta, GA, USA) was used to establish a database, and all data were double-entered. All the data analyses were performed using STATA software, version 16.0 (STATA Corporation, College Station, TX, USA). Statistical significance was denoted by two-tailed $p$-values of $<0.05$.

\section{Results}

\section{Baseline Characteristics of the Study Population}

The background information of participants was displayed in Table 1. A total of 13,231 women of childbearing age were included for analysis. The average age of the women was $28.01 \pm 4.93$ years. The average parity was $2.30 \pm 0.65$.
Most participants, approximately $81.15 \%$, had achieved compulsory education-secondary school or above $(10,737)$. Approximately three-quarters $(74.64 \%)$ of the women were not employed or worked as farmers. Among the participants, $13.58 \%$ (1797) resided in urban areas, $27.56 \%$ (3647) had suffered from passive smoking, $61.38 \%(8121)$ had taken folic acid, $4.60 \%$ had taken iron, $62.76 \%$ (8304) had completed more than 5 periods of antenatal care, and $18.77 \%$ (2483) had taken medication during pregnancy. More than half $(56.85 \%)$ of the infants were males. The weighted prevalence of LBW, SGA, PTB, and birth defects were $3.24 \%, 12.96 \%$, $2.93 \%$, and $2.12 \%$, respectively. Table 1 shows the prevalence of four adverse pregnancy outcomes in the groups.

\section{The Prevalence of Adverse Birth Outcomes Associated with Various IPIs}

Among the participants, $10.39 \%$ (1375) and $11.00 \%$ (1455) had IPIs of $<6$ or $>120$ months, respectively. The participants with IPIs of 60-119 months accounted for $24.28 \%$. The average IPI was $39.77(15.76,79.77)$ months. There were significant differences in the prevalence of LBW, SGA, PTB, and birth defects across the IPI groups $(\mathrm{P}<0.05)$ (Table 2).

\section{Association Between IPI and Adverse Pregnancy Outcomes}

After adjusting for potential confounding factors, the GLM showed that an IPI of $<6$ months increased the risks of SGA ( $R R=1.25,95 \%$ CI: 1.04-1.52) and birth defects ( $R R=2.55,95 \%$ CI: $1.45-4.47$ ), and IPIs of $\geq 120$ months were associated with higher risks of $\mathrm{LBW}(\mathrm{RR}=1.54,95 \%$ CI: 1.01-2.34) and PTB (RR=1.73, 95\% CI: 1.08-2.76) than IPIs of 18-23 months. Compared with the reference, IPIs of 6-11 months (RR=2.14, 95\% CI: $1.20-3.84)$ were associated with a higher risk of birth defects (Table 3).

\section{The Dose-Response Relationship Between IPI and Adverse Pregnancy Outcomes}

Figure 2 showed the dose-response relationships between IPI and the adverse pregnancy outcomes using RCS functions. There were nonlinear associations between IPI and LBW ( $\mathrm{p}$ for nonlinear association $=0.001, \mathrm{P}$ for overall association $<0.001$ ), SGA ( $p$ for nonlinear association $<0.001$, P for overall association $<0.001$ ), PTB ( $p$ for 
Table I Characteristics of the Participants in Northwest China During 2010-2013

\begin{tabular}{|c|c|c|c|c|c|}
\hline \multirow[t]{2}{*}{ Characteristics } & \multirow[t]{2}{*}{$\mathbf{N}$} & LBW & SGA & PTB & Birth Defects \\
\hline & & $468(3.54)$ & $1910(14.44)$ & $368(2.78)$ & $315(2.38)$ \\
\hline \multicolumn{6}{|l|}{ Childbearing age (year) } \\
\hline$<25$ & $3410(25.77)$ & $119(3.49)$ & $522(15.31)$ & $86(2.52)$ & $86(2.52)$ \\
\hline $25-29$ & $5603(42.35)$ & $193(3.44)$ & $801(14.30)$ & $156(2.78)$ & $125(2.23)$ \\
\hline $30-34$ & $2691(20.34)$ & $97(3.60)$ & $356(13.23)$ & $76(2.82)$ & $67(2.49)$ \\
\hline$\geq 35$ & I527 (I I.54) & $59(3.86)$ & $231(15.13)$ & $50(3.27)$ & $37(2.42)$ \\
\hline \multicolumn{6}{|l|}{ Maternal education } \\
\hline Primary school or lower & $2494(18.85)$ & $130(5.21)^{*}$ & $490(19.65)^{*}$ & $69(2.77)$ & $60(2.4 I)$ \\
\hline Secondary school & 7581 (57.30) & $252(3.32)$ & $1105(14.58)$ & $204(2.69)$ & $184(2.43)$ \\
\hline Senior high school or higher & $3156(23.85)$ & $86(2.72)$ & $315(9.98)$ & $95(3.01)$ & 7I (2.25) \\
\hline \multicolumn{6}{|l|}{ Residence } \\
\hline Rural & II,434 (86.42) & $426(3.73)^{*}$ & $1750(|5.3|)^{*}$ & $311(2.72)$ & $279(2.44)$ \\
\hline Urban & $1797(13.58)$ & $42(2.34)$ & $160(8.90)$ & $57(3.17)$ & $36(2.00)$ \\
\hline \multicolumn{6}{|l|}{ Family economic status } \\
\hline Low & $3010(22.75)$ & I 17 (3.89) & $446(14.82)^{*}$ & 81 (2.69) & $80(2.66)$ \\
\hline Middle & $7376(55.75)$ & $271(3.67)$ & $1119(15.17)$ & $214(2.90)$ & $165(2.24)$ \\
\hline High & $2845(21.50)$ & $80(2.8 I)$ & $345(12.13)$ & $73(2.57)$ & $70(2.46)$ \\
\hline \multicolumn{6}{|l|}{ Maternal Occupation } \\
\hline Peasant/housework & $9875(74.64)$ & $377(3.82)^{*}$ & $1532(|5.5|)^{*}$ & $266(2.69)$ & $243(2.46)$ \\
\hline Others & $3356(25.36)$ & $91(2.71)$ & $378(11.26)$ & $102(3.04)$ & $72(2.15)$ \\
\hline \multicolumn{6}{|l|}{ Passive smoking } \\
\hline Yes & $3647(27.56)$ & $138(3.78)$ & $563(15.44)^{*}$ & $100(2.74)$ & $118(3.24)^{*}$ \\
\hline No & $9584(72.44)$ & $330(3.44)$ & $1347(14.05)$ & $268(2.80)$ & $197(2.06)$ \\
\hline \multicolumn{6}{|l|}{ Folic Acid Supplement } \\
\hline Yes & $8121(61.38)$ & $247(3.04)^{*}$ & $1042(12.83)^{*}$ & $211(2.60)$ & $183(2.25)$ \\
\hline No & $5110(38.62)$ & $221(4.32)$ & $868(16.99)$ & $157(3.07)$ & $132(2.58)$ \\
\hline \multicolumn{6}{|l|}{ Iron Supplement } \\
\hline Yes & $608(4.60)$ & $13(2.14)$ & $59(9.70)^{*}$ & $17(2.80)$ & $6(0.99)^{*}$ \\
\hline No & $12,623(95.40)$ & $455(3.60)$ & $1851(14.66)$ & $35 \mathrm{I}(2.78)$ & $309(2.45)$ \\
\hline \multicolumn{6}{|l|}{ Frequency of antenatal care visits } \\
\hline$\geq 5$ & $8304(62.76)$ & $249(3.00)^{*}$ & $1030(12.40)^{*}$ & $237(2.85)$ & $193(2.32)$ \\
\hline$<5$ & $4927(37.24)$ & $219(4.44)$ & $880(17.86)$ & $|3|(2.66)$ & $122(2.48)$ \\
\hline \multicolumn{6}{|l|}{ Maternal taking medicine } \\
\hline Yes & $2483(18.77)$ & $112(4.5 \mathrm{I})^{*}$ & $360(14.50)$ & $98(3.95)^{*}$ & II 4 (4.59)* \\
\hline No & $10,748(81.23)$ & $356(3.31)$ & $1550(\mid 4.42)$ & $270(2.5 \mathrm{I})$ & $201(1.87)$ \\
\hline \multicolumn{6}{|l|}{ Infant gender } \\
\hline Male & $7522(56.85)$ & $221(2.94)^{*}$ & $1044(13.88)^{*}$ & $214(2.84)$ & 195 (2.59) \\
\hline Female & $5709(43.15)$ & $247(4.33)$ & $866(15.17)$ & $154(2.70)$ & $120(2.10)$ \\
\hline
\end{tabular}

Notes: The variables are presented as $\mathrm{n}(\%) ;{ }^{*} \mathrm{P}<0.05$.

Abbreviations: LBW, low birth weight; SGA, small for gestational age; PTB, preterm birth.

nonlinear association $=0.023, \mathrm{P}$ for overall association $<0.001$ ) and birth defects ( $p$ for nonlinear association $=0.007, \mathrm{P}$ for overall association $<0.001$ ), respectively. The J-shape curves were provided in Figure 2A-C, which indicated that the risk of adverse pregnancy outcomes increased with the short and long IPIs. Figure 2D showed that there was an inversely nonlinear association between IPI and birth defects. 
Table 2 Prevalence of Adverse Birth Outcomes at Various IPIs in Northwest China

\begin{tabular}{|l|l|l|l|l|l|}
\hline IPI (Months) & N & LBW* & SGA* & PTB* & Birth Defects* \\
\hline$<6$ & $1375(10.39)$ & $55(4.00)$ & $236(17.16)$ & $38(2.76)$ & $55(4.00)$ \\
$6-11$ & $1242(9.39)$ & $49(3.95)$ & $176(14.17)$ & $31(2.50)$ & $42(3.38)$ \\
$12-17$ & $1120(8.46)$ & $36(3.21)$ & $145(12.95)$ & $34(3.04)$ & $28(2.50)$ \\
$18-23$ & $951(7.19)$ & $29(3.05)$ & $136(14.30)$ & $24(2.52)$ & $15(1.58)$ \\
$24-59$ & $3876(29.29)$ & $110(2.84)$ & $519(13.39)$ & $91(2.35)$ & $85(2.19)$ \\
$60-119$ & $3212(24.28)$ & $113(3.52)$ & $443(13.79)$ & $90(2.80)$ & $65(2.02)$ \\
$\geq 120$ & $1455(11.00)$ & $76(5.22)$ & $255(17.53)$ & $60(4.12)$ & $25(1.72)$ \\
\hline
\end{tabular}

Notes: The variables are presented as $\mathrm{n}(\%) ; \mathrm{P}<0.05$.

Abbreviation: IPI, interpregnancy interval.

Table 3 Association Between IPI and Adverse Pregnancy Outcomes

\begin{tabular}{|c|c|c|c|c|c|c|c|}
\hline & $<6$ & $6-11$ & $12-17$ & $18-23$ & 24-59 & $60-119$ & $\geq \mathbf{1 2 0}$ \\
\hline \multicolumn{8}{|l|}{ LBW } \\
\hline Model I & I.3I (0.84-2.04) & $1.29(0.82-2.03)$ & $1.05(0.65-1.71)$ & Ref. & $0.93(0.62-1.39)$ & $1.15(0.77-1.72)$ & $1.71(1.13-2.61)^{*}$ \\
\hline Model 2 & $1.37(0.88-2.13)$ & $1.36(0.87-2.13)$ & $1.04(0.65-1.69)$ & Ref. & $0.93(0.62-1.39)$ & I.I2 (0.75-I.68) & $1.54(1.01-2.34)^{*}$ \\
\hline \multicolumn{8}{|l|}{ SGA } \\
\hline Model I & $1.20(0.99-1.46)$ & $0.99(0.81-1.22)$ & $0.91(0.73-1.13)$ & Ref. & $0.94(0.79-1.12)$ & $0.96(0.8 I-I .15)$ & $1.23(1.01-1.48)^{*}$ \\
\hline Model 2 & $1.25(1.04-1.52)^{*}$ & $1.03(0.84-1.26)$ & $0.89(0.72-1.10)$ & Ref. & $0.93(0.78-1.10)$ & $0.93(0.78-I .12)$ & $1.12(0.92-1.35)$ \\
\hline \multicolumn{8}{|l|}{ PTB } \\
\hline Model I & $1.10(0.66-1.81)$ & $0.99(0.58-1.67)$ & I.2 (0.72-2.0I) & Ref. & $0.93(0.60-1.45)$ & I.II (0.7I-1.73) & $1.63(1.03-2.6)^{*}$ \\
\hline Model 2 & $1.10(0.66-1.82)$ & I.0I (0.6-I.7I) & $1.23(0.73-2.06)$ & Ref. & $0.95(0.6 \mathrm{I}-\mathrm{I} .49)$ & I.I5 (0.74-I.80) & $1.73(1.08-2.76)^{*}$ \\
\hline \multicolumn{8}{|l|}{ Birth defects } \\
\hline Model I & $2.54(1.44-4.46)^{*}$ & $2.14(1.20-3.84)^{*}$ & $1.59(0.85-2.95)$ & Ref. & $\mathrm{I} .39(0.8 \mathrm{I}-2.4)$ & $1.28(0.74-2.24)$ & $1.09(0.58-2.06)$ \\
\hline Model 2 & $2.55(1.45-4.47)^{*}$ & $2.21(1.23-3.96)^{*}$ & $1.61(0.86-2.99)$ & Ref. & I.44 (0.84-2.49) & $1.36(0.78-2.37)$ & $1.15(0.61-2.18)$ \\
\hline
\end{tabular}

Notes: $* \mathrm{P}<0.05$; Model I was unadjusted any covariates; Model 2 adjusted for the childbearing age, maternal education, residence, family economic status, maternal occupation, passive smoking, folic acid supplement, iron supplement, frequency of antenatal care visits, maternal taking medicine and the infant gender for LBW, SGA, PTB and birth defects, respectively.

Abbreviation: Ref., referent groups.

\section{Sensitivity Analysis}

To analyze whether the association between IPI and childbirth results changes with maternal age and iron supplement status, additional sensitivity analyses stratified by age group and iron supplement group were performed. The sensitivity analyses showed that an IPI of $<6$ months increased the risks of SGA ( $\mathrm{RR}=1.27,95 \% \mathrm{CI}: 1.01-$ 1.60) and birth defects ( $R R=2.16,95 \%$ CI: 1.11-4.22) among women aged $<35$. An IPI of $<6$ months increased the risks of birth defects ( $R R=3.54,95 \%$ CI: $1.22-10.25)$ and an IPI of $\geq 120$ months increased the risk of PTB $(\mathrm{RR}=3.07,95 \% \mathrm{CI}: 1.35-6.98)$ among women aged $\geq 35$ (Table S1). An IPI of $<6$ months increased the risks of SGA (RR $=1.27,95 \% \mathrm{CI}: 1.05-1.54)$ and birth defects $(\mathrm{RR}=2.52$, 95\% CI: $1.43-4.42)$ and an IPI of $\geq 120$ mons increased risks of LBW (RR=1.57, 95\% CI: 1.02-2.41) and PTB (RR=1.79, 95\% CI: 1.10-2.92) among women without iron supplementation during pregnancy. The associations between short and long IPIs and adverse birth outcomes were not statistically significant among women with iron supplementation during pregnancy. However, the RRs for associations between short and long IPIs and adverse birth outcomes among women with iron supplementation during pregnancy were lower than that among women without iron supplementation during pregnancy at any group (Table $\mathrm{S} 2)$.

\section{Discussion}

The present study aimed to reveal the relationship between the short and long IPIs and the adverse birth outcomes. We found that both short and long IPIs were associated with increased odds of adverse birth outcomes. Compared with the reference group, infants with a short IPI ( $<6$ months) had higher risks of SGA and birth defects and those with 

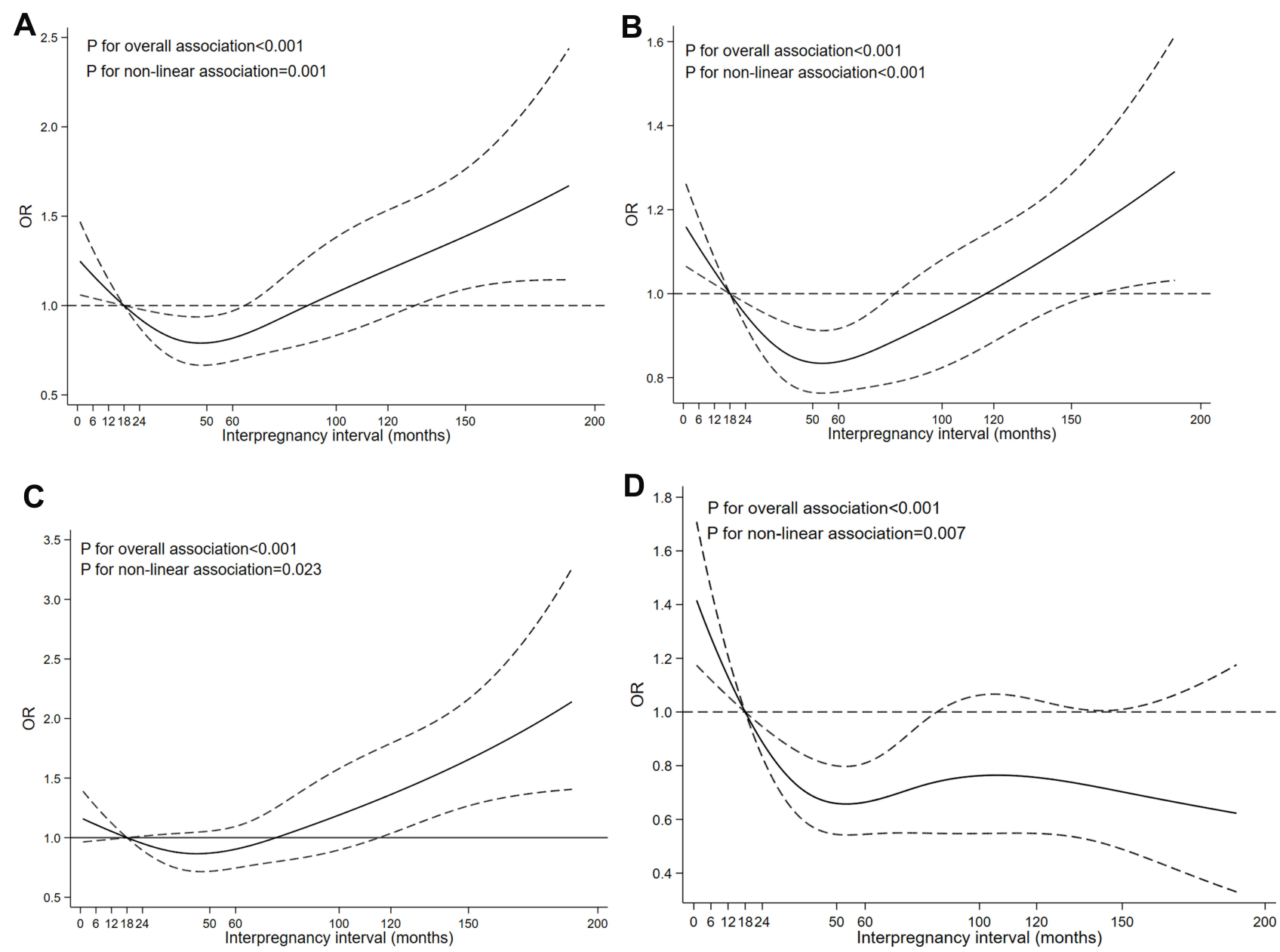

Figure 2 Dose-response relationships between IPI and LBW, SGA, PTB and birth defects.

Notes: (A) Association between IPI and LBW using restricted cubic spline functions. (B) Association between IPI and SGA using restricted cubic spline functions. (C) Association between IPI and PTB using restricted cubic spline functions. (D) Association between IPI and birth defects using restricted cubic spline functions. The childbearing age, maternal education, residence, family economic status, maternal occupation, passive smoking, folic acid supplement, iron supplement, frequency of antenatal care visits, maternal taking medicine and the infant gender were adjusted for LBW, SGA, PTB and birth defects, respectively.

a long IPI ( $>12$ months) had higher risks of LBW and PTB. In addition, the study suggested significant doseresponse relationships between IPI and adverse birth outcomes (including LBW, SGA, PTB, and birth defects).

The association between short IPIs (typically defined as $<6$ months) and adverse perinatal outcomes had been explored in previous studies. ${ }^{17,29,30}$ A study conducted by Schummers et al in a Canadian population had proved that an IPI of $<6$ months contributes to the higher risk of SGA. ${ }^{17}$ Kwon et al found that women with short IPIs had an increased risk of birth defects. ${ }^{30}$ A previous study reported short IPIs ( $<6$ months) increased the risk of PTB. ${ }^{16}$ Our results confirm the above research. In addition, some studies had reported inverse results. Ball et al investigated the association between IPIs and birth outcomes and showed that short IPIs had little effect on the incidence of PTB and LBW. They proposed that the association between short IPIs and adverse birth outcomes in previous research may be attributable to unassessed confounding factors, such as unplanned pregnancies, fertility, family/social disruptions, and maternal illness. ${ }^{20}$ Gebremedhin et al reported that the associations between short IPIs and hypertensive disorders of pregnancy and gestational diabetes were not statistically significant based on within-mother matched analyses and suggested that associations observed in previous studies may have been due to confounding factors. ${ }^{31,32}$ Due to the limitations of the pre-set questions, several influencing factors had not been included in this study. Studies with better investigations and better designs will be needed in the future to analyze the correlation between IPI and adverse birth outcomes. Several hypotheses have been proposed to 
explain the adverse birth outcomes associated with short IPIs. A popular explanation was that the mother may not have enough time to recover from nutritional deficits due to the stress of the first pregnancy and subsequent breastfeeding. The second pregnancy would intensify the competition between mothers and fetuses for essential nutrients. ${ }^{33,34}$ For example, insufficient supplementation for the physiologic consumption of iron caused by pregnancy or breastfeeding may lead to iron deficiency in future pregnancies, which can increase the risk of LBW and PTB. ${ }^{35,36}$ The sensitivity analyses showed that the RRs for associations between short IPI and adverse birth outcomes among women with iron supplementation during pregnancy were lower than that among women without iron supplementation during pregnancy at any group which further confirmed the hypothesis.

Previous studies exploring the effect of IPI on adverse birth outcomes mainly focused on short IPIs, while long IPIs had received far less attention. This study investigated the association between long IPIs and adverse birth outcomes and suggested that infants with mothers who had long IPIs ( $>120$ months) had an increased risk of LBW and PTB. Lin et al found that women with IPIs of $>120$ months had significantly increased risks of gestational diabetes mellitus, gestational hypertension, and premature membrane rupture than those with IPIs of 12-23 months. ${ }^{13}$ A previous investigation conducted in Bangladesh reported that IPIs of $\geq 60$ months were associated with higher risks of small birth size and first-day neonatal deaths than IPIs of 36-59 months. ${ }^{5}$ These findings were consistent with our results. This association had been questioned in some studies. Hanley et al showed that the associations of gestational diabetes mellitus and preeclampsia with long IPIs were eliminated by using withinwoman matched analyses. ${ }^{37}$ Ball et al also reported similar results. ${ }^{20}$ We need to interpret the relationships between long birth intervals and pregnancy outcomes cautiously in clinical practice because of the rareness of studies on this issue and the inconsistency of results. China announced the implementation of a comprehensive two-child policy and abolished the one-child policy in $2015 .^{38}$ A retrospective study conducted in China found that women with IPIs of $>96$ months accounted for $21.3 \%$, ${ }^{39}$ which indicated that there was a large proportion of women with long IPIs in China. Our study found that women with IPIs of $>120$ months accounted for $10 \%$ between 2010 and 2013. After the implementation of the two-child policy, several couples who previously had only one child would have a second child, which could result in more women having long IPIs. Therefore, the adverse birth outcomes associated with longer IPIs should arouse more attention in China. The mechanism underlying the association between long IPIs and poor birth outcomes has not been established; however, the "physiological regression hypothesis" may explain it. The hypothesis suggests that mothers may have adaptive benefits from pregnancy and the benefits may gradually weaken after delivery until their physiological characteristics return to the same state as primigravida. ${ }^{9,34}$

Moreover, our study also found obvious dose-response relationships between IPI and LBW, SGA, PTB and birth defects. Consistent with previous studies, ${ }^{9,17}$ the J-shape curves were observed for the relationships between IPI and LBW and SGA and PTB, which also further confirmed the results of GLM. However, there was no J-shape relationship between IPI and birth defects, which was different from the findings of previous studies. ${ }^{12}$ Different population characteristics and adjustment factors may account for this difference. We found that the lowest risks of the four adverse birth outcomes were associated with an IPI of approximately 50 months, as shown in Figure 2A-D. Several studies had suggested IPIs of 27-50 months as optimal. ${ }^{5,40,41}$ Further studies with a favorable design were needed to verify the optimal birth interval. Nevertheless, both the GLM and dose-response relationship found short and long IPIs associated with a high risk of adverse outcomes. Maternal and pregnancy characteristics (such as obesity and gestational diabetes) related to IPIs may vary with maternal age, ${ }^{42,43}$ which may affect the association between IPI and adverse birth outcomes to some extent. Some studies focused on this issue, ${ }^{17,44}$ but they were limited. To verify the results above and assess whether the association between IPIs and birth outcomes varies with maternal age, we performed a sensitivity analysis that suggested that the adverse pregnancy outcomes associated with IPIs were applicable to women of all ages.

The main strength of this study was that it involved a large representative sample from a stratified multistage random sampling survey. Considering the similarities in the cultural distribution, economy, and lifestyle in Northwest China, our results can be generalized for Northwest China to some extent. Besides, a multistatistical approach involving dose-response relationships and sensitivity analyses were performed to verify the results. In addition, the birth outcomes gathered through a review of medical records and birth certificates 
were relatively accurate. Moreover, several limitations should be stated. First of all, due to the limitations of the pre-set questions, indicators, such as intrauterine growth restriction, were not included as outcome variables, which restricted our exploration. Secondly, residual confounding caused by some unassessed factors (such as pre-pregnancy body mass index, weight gain during pregnancy, fertility, and maternal illness) may affect the results, and this could lead to a spurious association. Furthermore, this study used a cross-sectional design, and the characteristics of the participants were retrospectively self-reported, and recall bias could not be avoided although we had taken steps to help control recall bias, including using a standard questionnaire to collect the data, training interviewers rigorously, and conducting a pilot study to test the survey instruments.

\section{Conclusion}

The present study suggests independent associations between short and long IPIs and the risks of adverse birth outcomes. Our findings provide evidence in support of family planning, for both patients and health care providers. Further well-designed studies are needed to explore these associations and explain the underlying mechanisms.

\section{Data Sharing Statement}

The data used and/or analyzed during the study are available from the corresponding author's (SD or HY) on reasonable request.

\section{Ethics Approval and Consent to Participate}

This study was approved by the Medical Ethics Committee of Xi'an Jiaotong University (No. 20112008). There were no participants under 18 years of age and information about newborns was provided by their mothers. Written informed consent was obtained from all participants prior to questionnaire administration. This study was performed in accordance with the ethical standards of the Declaration of Helsinki.

\section{Acknowledgments}

The authors would like to thank all the families in the study and all the investigators. We are also grateful to the local hospitals, health administrative departments and the Ministry of Health in Shaanxi province for their support in coordinating field work and data collection. This study was supported by National Natural Science Foundation of China
(No. 81230016), Shaanxi Health and Family Planning Commission (No. Sxwsjswzfcght2016-013), the Natural Science Basic Research Plan of the Shaanxi Province (No. 2020JM-077) and National Key R\&D Program of China (No. 2017YFC0907200, 2017YFC0907201). These sponsors had no role in the design, data collection, and writing of this manuscript.

\section{Disclosure}

The authors report no conflicts of interest in this work.

\section{References}

1. Gluckman PD, Hanson MA, Cooper C, Thornburg KL. Effect of in utero and early-life conditions on adult health and disease. $N$ Eng J Med. 2008;359(1):61-73. doi:10.1056/NEJMra0708473

2. Collaborators GS. Measuring progress and projecting attainment on the basis of past trends of the health-related sustainable development goals in 188 countries: an analysis from the Global Burden of Disease Study 2016 (vol 390, pg 1423, 2017). Lancet. 2017;390 (10106):E38.

3. Blencowe H, Cousens S, Oestergaard MZ, et al. National, regional, and worldwide estimates of preterm birth rates in the year 2010 with time trends since 1990 for selected countries: a systematic analysis and implications. Lancet. 2012;379(9832):2162-2172. doi:10.1016/ S0140-6736(12)60820-4

4. Walani SR. Global burden of preterm birth. Int J Gynaecol Obstet. 2020;150(1):31-33. doi:10.1002/ijgo.13195

5. Nisha MK, Alam A, Islam MT, Huda T, Raynes-Greenow C. Risk of adverse pregnancy outcomes associated with short and long birth intervals in Bangladesh: evidence from six Bangladesh demographic and health surveys, 1996-2014. BMJ Open. 2019;9(2):e024392. doi:10.1136/bmjopen-2018-024392

6. Qu P, Li S, Liu D, et al. A propensity-matched study of the association between optimal folic acid supplementation and birth defects in Shaanxi province, Northwestern China. Sci Rep. 2019;9:1-9. doi:10.1038/s41598-019-41584-5

7. Chen YH, Li L, Chen W, et al. Pre-pregnancy underweight and obesity are positively associated with small-for-gestational-age infants in a Chinese population. Sci Rep. 2019;9:1-8. doi:10.1038/ s41598-019-52018-7

8. Guo L, Qu P, Zhang R, et al. Propensity score-matched analysis on the association between pregnancy infections and adverse birth outcomes in rural northwestern China. Sci Rep. 2018;8:1-8. doi:10.1038/ s41598-018-23306-5

9. Conde-Agudelo A, Rosas-Bermudez A, Kafury-Goeta AC. Birth spacing and risk of adverse perinatal outcomes - A meta-analysis. JAMA- J Am Med Assoc. 2006;295(15):1809-1823. doi:10.1001/ jama.295.15.1809

10. Wendt A, Gibbs CM, Peters S, Hogue CJ. Impact of increasing inter-pregnancy interval on maternal and infant health. Paediatr Perinat Epidemiol. 2012;26:239-258. doi:10.1111/j.13653016.2012.01285.x

11. Shachar BZ, Lyell DJ. Interpregnancy interval and obstetrical complications. Obstet Gynecol Surv. 2012;67(9):584-596. doi:10.1097/OGX.0b013e31826b2c3e

12. Zhu BP. Effect of interpregnancy interval on birth outcomes: findings from three recent US studies. Int J Gynaecol Obstet. 2005;89:S25S33. doi:10.1016/j.ijgo.2004.08.002

13. Lin J, Liu H, Wu DD, et al. Long interpregnancy interval and adverse perinatal outcomes: a retrospective cohort study. Sci China Life Sci. 2020;63(6):898-904. doi:10.1007/s11427-018-9593-8 
14. Worl Health Organization. Report of a WHO technical consultation on birth spacing: Geneva, Switzerland 13-15 June 2005. 2007.

15. Grisaru-Granovsky S, Gordon ES, Haklai Z, Samueloff A, Schimmel MM. Effect of interpregnancy interval on adverse perinatal outcomes - a national study. Contraception. 2009;80(6):512-518. doi:10.1016/j.contraception.2009.06.006

16. Lonhart JA, Mayo JA, Padula AM, Wise PH, Stevenson DK, Shaw GM. Short interpregnancy interval as a risk factor for preterm birth in non-hispanic black and white women in California. J Perinatol. 2019;39(9):1175-1181. doi:10.1038/s41372-019-0402-1

17. Schummers L, Hutcheon JA, Hernandez-Diaz S, et al. Association of short interpregnancy interval with pregnancy outcomes according to maternal age. JAMA Intern Med. 2018;178(12):1661-1670. doi:10.1001/jamainternmed.2018.4696

18. Zhang Q, Wang LL, Bai RH, Dang SN, Yan H. Effect of interpregnancy interval of childbearing aged women on birth weight of single live birth neonates. Zhonghua Liu Xing Bing Xue Za Zhi = Zhonghua Liuxingbingxue Zazhi. 2018;39(3):317-321. doi:10.3760/cma.j. issn.0254-6450.2018.03.013

19. Conde-Agudelo A, Belizan JM, Norton MH, Rosas-Bermudez A. Effect of the interpregnancy interval on perinatal outcomes in Latin America. Obstet Gynecol. 2005;106(2):359-366. doi:10.1097/01. AOG.0000171118.79529.a3

20. Ball SJ, Pereira G, Jacoby P, de Klerk N, Stanley FJ. Re-evaluation of link between interpregnancy interval and adverse birth outcomes: retrospective cohort study matching two intervals per mother. $B M J$ Br Med J. 2014;349. doi:10.1136/bmj.g4333

21. Zhu L, Zhang R, Zhang S, et al. [Chinese neonatal birth weight curve for different gestational age]. Zhonghua $\mathrm{Er} \mathrm{Ke} \mathrm{Za} \mathrm{Zhi=Chin}$ J Pediatr. 2015;53(2):97-103. [Chinese].

22. Pei L, Kang Y, Cheng Y, Yan H. The association of maternal lifestyle with birth defects in Shaanxi Province, Northwest China. PLoS One. 2015;10(9):e0139452. doi:10.1371/journal.pone.0139452

23. Bazyar J, Daliri S, Sayehmiri K, Karimi A, Delpisheh A. Assessing the relationship between maternal and neonatal factors and low birth weight in Iran; a systematic review and meta-analysis. J Med Life. 2015;8(SpecIss 4):23-31.

24. Chawanpaiboon S, Vogel JP, Moller AB, et al. Global, regional, and national estimates of levels of preterm birth in 2014: a systematic review and modelling analysis. Lancet Global Health. 2019;7(1): E37-E46. doi:10.1016/S2214-109X(18)30451-0

25. Harris BS, Bishop KC, Kemeny HR, Walker JS, Rhee E, Kuller JA. Risk factors for birth defects. Obstet Gynecol Surv. 2017;72 (2):123-135. doi:10.1097/ogx.0000000000000405

26. Guo L, Zhao D, Zhang R, et al. A matched case-control study on the association between colds, depressive symptoms during pregnancy and congenital heart disease in Northwestern China. Sci Rep. 2019;9 (1): $1-11$

27. Yang G, Fan L, Tan J, et al. Smoking in China: findings of the 1996 national prevalence survey. JAMA. 1999;282(13):1247-1253. doi:10.1001/jama.282.13.1247

28. Zhang R, Li C, Mi B, et al. The different effects of prenatal nutrient supplementation on neonatal birth weights between urban and rural areas of northwest China: a cross-sectional study. Asia Pac J Clin Nutr. 2018.

29. Mignini LE, Carroli G, Betran AP, et al. Interpregnancy interval and perinatal outcomes across Latin America from 1990 to 2009: a large multi-country study. BJOG-Int J Obstet Gynaecol. 2016;123 (5):730-737. doi:10.1111/1471-0528.13625
30. Kwon S, Lazo-Escalante M, Villaran MV, Li CI. Relationship between interpregnancy interval and birth defects in Washington State. J Perinatol. 2012;32(1):45-50. doi:10.1038/jp.2011.49

31. Gebremedhin AT, Regan AK, Ball S, et al. Interpregnancy interval and hypertensive disorders of pregnancy: a population-based cohort study. Paediatr Perinat Epidemiol. 2020. doi:10.1111/ppe.12668

32. Gebremedhin AT, Regan AK, Ball S, et al. Effect of interpregnancy interval on gestational diabetes: a retrospective matched cohort study. Ann Epidemiol. 2019;39:33-38. doi:10.1016/j.annepidem.20 19.09.004

33. Zhu BP, Rolfs RT, Nangle BE, Horan JM. Effect of the interval between pregnancies on perinatal outcomes. $N$ Eng $J$ Med. 1999;340(8):589-594. doi:10.1056/nejm199902253400801

34. Marinovich ML, Regan AK, Gissler $M$, et al. Developing evidence-based recommendations for optimal interpregnancy intervals in high-income countries: protocol for an international cohort study. BMJ Open. 2019;9(1):e027941. doi:10.1136/bmjopen-2018027941

35. Yang J, Cheng Y, Pei L, et al. Maternal iron intake during pregnancy and birth outcomes: a cross-sectional study in Northwest China. $\mathrm{Br}$ J Nutr. 2017;117(6):862-871. doi:10.1017/s0007114517000691

36. Liu D, Cheng Y, Dang S, et al. Maternal adherence to micronutrient supplementation before and during pregnancy in Northwest China: a large-scale population-based cross-sectional survey. BMJ Open. 2019;9(8):e028843. doi:10.1136/bmjopen-2018-028843

37. Hanley GE, Hutcheon JA, Kinniburgh BA, Lee L. Interpregnancy interval and adverse pregnancy outcomes an analysis of successive pregnancies. Obstet Gynecol. 2017;129(3):408-415. doi:10.1097/ aog.0000000000001891

38. Zeng Y, Hesketh T. The effects of China's universal two-child policy. Lancet. 2016;388(10054):1930-1938. doi:10.1016/S0140-6736(16) 31405-2

39. Qin C, Mi C, Xia A, et al. A first look at the effects of long inter-pregnancy interval and advanced maternal age on perinatal outcomes: a retrospective cohort study. Birth-Iss Perinat Care. 2017;44 (3):230-237. doi:10.1111/birt.12289

40. Exavery A, Mrema S, Shamte A, et al. Levels and correlates of non-adherence to WHO recommended inter-birth intervals in Rufiji, Tanzania. BMC Pregnancy Childbirth. 2012;12:12152. doi:10.1186/ 1471-2393-12-152

41. Rutstein SO. Effects of preceding birth intervals on neonatal, infant and under-five years mortality and nutritional status in developing countries: evidence from the demographic and health surveys. Int $J$ Gynaecol Obstet. 2005;89:S7-S24. doi:10.1016/j.ijgo.20 04.11 .012

42. Finer LB, Zolna MR. Declines in unintended pregnancy in the United States, 2008-2011. N Eng J Med. 2016;374(9):843-852. doi:10.1056/ NEJMsa 1506575

43. Lean SC, Derricott H, Jones RL, Heazell AEP. Advanced maternal age and adverse pregnancy outcomes: a systematic review and meta-analysis. PLoS One. 2017;12(10):e0186287. doi:10.1371/journal.pone. 0186287

44. De Weger FJ, Hukkelhoven CWPM, Serroyen J, Velde ERT, Smits LJM. Advanced maternal age, short interpregnancy interval, and perinatal outcome. Am J Obstet Gynecol. 2011;204(5):421-e1. doi:10.1016/j.ajog.2010.12.008 


\section{Publish your work in this journal}

The International Journal of General Medicine is an international, peer-reviewed open-access journal that focuses on general and internal medicine, pathogenesis, epidemiology, diagnosis, monitoring and treatment protocols. The journal is characterized by the rapid reporting of reviews, original research and clinical studies

across all disease areas. The manuscript management system is completely online and includes a very quick and fair peer-review system, which is all easy to use. Visit http://www.dovepress.com/ testimonials.php to read real quotes from published authors.

Submit your manuscript here: https://www.dovepress.com/international-journal-of-general-medicine-journal 